\title{
Does a Simple Hand Grip Strength Test Prognosticate Postoperative Pneumonia Risk Following Esophagectomy?
}

\author{
Dana McCloskey, MD ${ }^{1}$, and David D. Shersher, MD ${ }^{2}$ \\ ${ }^{1}$ Department of Surgery, Cooper University Hospital, Camden, NJ; ${ }^{2}$ Department of Surgery, Division of Thoracic Surgery, \\ Cooper MD Anderson Cancer Center, Cooper University Hospital, Camden, NJ
}

Even in the most skilled hands, morbidity following esophagectomy for esophageal cancer remains high, with pneumonia being the most prominent serious morbidity, occurring in $7.3-38 \%$ of cases. ${ }^{1,2}$ Pulmonary complications have been associated with up to $55 \%$ of inpatient deaths following esophagectomy and are associated with increased length of stay, hospital cost, and the incidence of alternative postoperative complications., ${ }^{2,3}$ Preoperative predictive models for postoperative complications following esophagectomy are scarce, making it difficult for surgeons to predict accurately and easily the perioperative risk in patients with tumors otherwise amenable to surgical resection. Small-scale studies have identified notable risk factors that may increase risk for postoperative pulmonary complications, including advanced age, preexisting pulmonary dysfunction, tobacco use, inadequate nutritional status, performance status, tumor characteristics, and operative factors, such as operative time. ${ }^{1,3}$ These factors, however, are not easily modifiable to ameliorate postoperative risk.

In an effort to mitigate postoperative comorbidity following esophagectomy, and specifically postoperative pulmonary complications, identification of modifiable risk factors, particularly in the preoperative setting, is vital.

Editorial on "Handgrip Strength Predicts Postoperative Pneumonia after Thoracoscopic-Laparoscopic Esophagectomy in Patients with Esophageal Cancer" by Daiko, Hiroyuki et al. (ASO-2020-03-0552).

(C) Society of Surgical Oncology 2020

First Received: 14 April 2020;

Published Online: 30 April 2020

D. D. Shersher, MD

e-mail: Shersher-david@cooperhealth.edu
Although there are no published reports to establish the effectiveness of preoperative pulmonary physiotherapy in patients undergoing esophagectomy for esophageal cancer, one may infer that improving perioperative exercise capacity or lung function in this population would reduce pulmonary complications based on existing evidence. Preoperative chest physiotherapy has been employed with success following lung resection, coronary artery bypass graft (CABG), and major abdominal surgery as a means to improve functional ability and reduce postoperative morbidity. ${ }^{4-6}$ Along with smoking cessation, patient participation in preoperative pulmonary rehabilitation programs for a duration of up to 3 weeks contributed to significant improvements in pulmonary function, inspiratory muscle function, respiratory muscle endurance, exercise capacity, and muscle quality. ${ }^{4,5}$ In patients undergoing $\mathrm{CABG}$, nearly a $50 \%$ risk reduction in postoperative pulmonary complications may be observed following implementation of prophylactic tailored inspiratory muscle therapy. ${ }^{5}$

The question remains: which patient should be targeted for preoperative physiotherapy, particularly following esophagectomy for malignancy? Algorithms and scoring systems to evaluate pulmonary risk and overall risk of postoperative morbidity are sparse and not sufficiently accurate for widespread use. ${ }^{3,7}$ Objective modifiable preoperative markers of postoperative pulmonary complications have yet to be identified in this population. What appears clinically obvious in a high-risk population group, such as esophageal cancer patients undergoing trimodality therapy, is not supported by good, randomized, controlled data.

Sarcopenia as defined by presence of low muscle mass and low muscle function may be a reproducible metric to prognosticate outcome. ${ }^{8}$ More so than in other cancer, 
skeletal muscle mass is known to be reduced in patients with esophageal cancer, because it is invariably associated with malnutrition due to dysphagia, inanition, and profound weight loss. ${ }^{89}$ Sarcopenia is becoming increasingly recognized as the physiologic manifestation of fasting, malnutrition, and cachexia, which itself has been associated with poor prognosis. ${ }^{89}$ This is particularly true in elderly patients, who in general tend to have higher rates of comorbidities and decreased skeletal muscle mass with age. ${ }^{8,9}$ With respect to survival in the elderly, sarcopenia is as important prognostically as tumor characteristics, including tumor depth (T stage). ${ }^{9,10}$ When stratifying for age, sarcopenia is an independent prognostic factor for anastomotic leak and overall survival in elderly patients with esophageal cancer. ${ }^{9}$ Prognosis overall is worse in patients with greater postoperative skeletal muscle index (SMI) loss compared with those with mild SMI loss. ${ }^{10}$ Both the presence of sarcopenia before surgery, and the degree to which sarcopenia worsens after surgery, are associated with worse overall prognosis in cases of esophagectomy for esophageal cancer. ${ }^{10}$

From the respiratory perspective, patients with sarcopenia have decreased pulmonary function compared with patients with preserved muscle function and quantity. ${ }^{8}$ Sarcopenia is an independent risk factor for postoperative pulmonary complications and in some reports is associated with a 2.6 relative risk of developing postoperative pulmonary complications compared with patients without sarcopenia. ${ }^{1,8}$ Sarcopenia influences both preoperative respiratory function and postoperative respiratory complications, potentially through decreased muscle strength of respiratory and pharyngeal muscles or the increased inflammatory effect observed in sarcopenic individuals, or both. ${ }^{1,8}$ Perhaps specific to esophagectomy, an additional hypothesis is that sarcopenia is associated with cachexia and, when coupled with morbid surgery, correlates to reduced pulmonary effort, cough suppression from pain, and ultimately the inability to clear secretions adequately, leading to pneumonia.

Given the profound effect of sarcopenia on postoperative outcomes following esophagectomy, including pulmonary complications, it represents a high-yield target for improving functional ability before surgery. Measurements preoperatively may aid to identify patients who may benefit from preoperative rehabilitation, nutritional support, or those with high postoperative risk in which esophagectomy may be prohibited. However, establishing sarcopenia as a reliable and quantitative component of preoperative risk stratification has been challenging due to ambiguity in data collection and to a lesser extent in consistent classification. Predominant studies include computed tomography measurements of SMI with varying cutoff values for sarcopenia and alternative techniques for assessment of skeletal muscle mass, including bioelectrical impedence or dual-energy X-ray absorptiometry, all of which may be challenging, time consuming, and ultimately unrealistic in the course of preoperative planning. ${ }^{1,8-10}$

We applaud Kurita et al. in their attempt to establish new, easily reproducible preoperative criteria that may stratify patients undergoing TLE into cohorts according to their risk of developing postoperative pneumonia, based on skeletal muscle function. The authors evaluated the use of hand grip strength (HGS) as a measure sarcopenia using the revised definition established by the European Working Group on Sarcopenia in Older People (EWGSOP2), specifically as a predictive factor for the development of pneumonia following esophagectomy.

This retrospective observational study from Japan included 161 men who underwent thoracoscopic-laparoscopic three-hole esophagectomy following neoadjuvant chemotherapy or radiotherapy at a single institution over a period of 2 years. HGS was measured following neoadjuvant treatment, with low-HGS $<27 \mathrm{~kg}$ and highHGS $\geq 27 \mathrm{~kg}$ according to EWGSOP2 definition, including $19(11.2 \%)$ and $142(88.2 \%)$ patients, respectively. Among the study population, $17.4 \%$ experienced postoperative pneumonia, $47.4 \%$ in the low-HGS group compared with $13.4 \%$ in the high-HGS group $(p \leq 0.001)$. Hospital length of stay additionally was longer in the low-HGS group compared with the high-HGS group, with mean of 16 days and 14 days, respectively $(p=0.001)$. Multivariate logistic regression analysis demonstrated that HGS was a significant predictive factor for postoperative pneumonia with odds ratio $(\mathrm{OR})$ of $1.21 \quad(p=0.010)$. Additional notable significant factors included postoperative laryngeal nerve palsy [OR, 3.84; confidence interval (CI) 1.03-11.30; $p=0.015$ ] and age [OR, 1.09; CI 1.00-1.19; $p=0.40$ ]. Preoperative SMI was calculated based on computer tomography at the third lumbar vertebral level and was found to have no association with incidence of postoperative pneumonia in the study group. Evaluating predictive accuracy for postoperative pneumonia, area under the receiving operating characteristic was higher for HGS compared with SMI $(0.79$ vs. 0.65 , respectively; $p=0.012$ ). The authors conclude HGS is a significant predictive factor of postoperative pneumonia after TLE for esophageal cancer.

One major limitation of the study is the heterogeneity of patient selection. The entirely male cohort inadequately represents the population of patients undergoing esophagectomy, although the female subgroup was too small to meaningfully include in the final analysis (29 women compared with 161 men). In a subgroup analysis of females, HGS was still significantly lower compared with their male counterparts (22.3 vs. 34.4 , respectively; $p$ $\leq 0.001$ ) with a greater proportion of women falling into 
the low-HGS classification. Although females in this study did not experience any postoperative pneumonia, it is difficult to interpret and compare due to the low number of patients in this cohort. Ultimately the authors decided to exclude this female group from data analysis, making it difficult to interpret low female HGS as it truly correlates to postoperative pneumonia after esophagectomy.

A second meaningful limitation also acknowledged by the author group is the inconsistency or inaccuracy of measurement of HGS, which should have been performed with patients resting in a seated position with forearms on the arms of the chair. Instead, this was measured with the patient in a standing position. It also is unclear whether there was consistency of examiner measuring these values, which could further add variability to the data. Finally, it would have been interesting to have another time point measure of HGS before initiation of any chemoradiotherapy neoadjuvant therapy, and not just within 30 days before esophagectomy, when many patients have already flattened the cachexia curve.

As more emphasis is placed on outcomes-based care to improve surgical safety and quality, the role of the data presented by Kurita et al. ${ }^{11}$ becomes increasingly important in preventative medicine. The need for cost-effective, benign, targeted therapies to improve postoperative outcomes and decrease overall healthcare expenditure is becoming an increasingly integral component of perioperative care. This is especially true in for high-risk surgical procedures, such as esophagectomy. Reproducible, accurate, and straightforward models of risk stratification are needed to aid in the allocation of healthcare resources to high-risk patients. Utilization of HGS as proposed by Kurita et al. ${ }^{11}$ is an efficient and benign method of assessing skeletal muscle function as a component of sarcopenia, which may be obtained at the time of surgical preoperative assessment. Its accuracy in predicting postoperative pneumonia following esophagectomy appears to be superior to SMI, which is still considered the used standard metric correlating pneumonia to sarcopenia in the surgical patient. HGS use as a solitary risk measure is still not validated across all groups but may add value when considered in conjunction with additional well-established measures of risk for post-esophagectomy morbidity, and particularly for pulmonary complications. We encourage and look forward to continued validation of the accuracy of this technique across a more diverse patient demographic, which should help to stratify "at risk" patients undergoing esophagectomy to additional rehabilitation measures designed specifically to reduce postoperative morbidity.

DISCLOSURE The authors declare no conflicts of interest.

\section{REFERENCES}

1. Nishigori T, et al. Sarcopenia as a predictor of pulmonary complications after esophagectomy for thoracic esophageal cancer. $J$ Surg Oncol. 2016;113(6):678-84.

2. Law S, et al. Predictive factors for postoperative pulmonary complications and mortality after esophagectomy for cancer. Ann Surg. 2004;240(5):791-800.

3. Ferguson MK, Celauro AD, Prachand V. Prediction of major pulmonary complications after esophagectomy. Ann Thorac Surg. 2011;91(5):1494-500; discussion 1500-1.

4. Vagvolgyi A, et al. Effectiveness of perioperative pulmonary rehabilitation in thoracic surgery. $J$ Thorac Dis. 2017;9(6):1584-91.

5. Hulzebos E, et al. Preoperative intensive inspiratory muscle training to prevent postoperative pulmonary complications in high-risk patients undergoing CABG surgery. JAMA. 2006;296(15):1851-7.

6. Boden I, et al. Preoperative physiotherapy for the prevention of respiratory complications after upper abdominal surgery: pragmatic, double blinded, multicentre randomised controlled trial. BMJ. 2018;360:5916.

7. Wright $\mathrm{CD}$, et al. Predictors of major morbidity and mortality after esophagectomy for esophageal cancer: a Society of Thoracic Surgeons General Thoracic Surgery Database risk adjustment model. J Thorac Cardiovasc Surg. 2009;137(3):587-95; discussion 596.

8. Ida S, et al. Sarcopenia is a predictor of postoperative respiratory complications in patients with esophageal cancer. Ann Surg Oncol. 2015;22(13):4432-7.

9. Nakashima Y, et al. Assessment of sarcopenia as a predictor of poor outcomes after esophagectomy in elderly patients with esophageal cancer. Ann Surg. 2018;267(6):1100-4.

10. Nakashima Y, et al. Skeletal muscle loss after esophagectomy is an independent risk factor for patients with esophageal cancer. Ann Surg Oncol. 2020;27(2):492-8.

11. Kurita D, et al. Handgrip strength predicts posoperative pneumonia after thoracoscopic-laparoscopic esophagectomy in patients with esophageal cancer. Ann Surg Oncol. 2020. https:// doi.org/10.1245/s10434-020-08520-8.

Publisher's Note Springer Nature remains neutral with regard to jurisdictional claims in published maps and institutional affiliations. 